\title{
Molecular study of ultrasound-triggered release of fluorescein from liposomes
}

Fatima El Hajj, ${ }^{\dagger, \ddagger}$ Patrick F. J. Fuchs, ${ }^{\nwarrow}$ Wladimir Urbach, ${ }^{\S}$ Mohammad Nassereddine, ${ }^{\ddagger}$ Salah Hamieh, ${ }^{\ddagger}$ and Nicolas Taulier*,†

† Sorbonne Université, CNRS, INSERM, Laboratoire d'Imagerie Biomédicale, LIB, F-75006Paris, France

${ }^{\ddagger}$ Faculté des Sciences, Université Libanaise, Liban

" Université de Paris

${ }^{\S}$ Laboratoire de Physique de l'École Normale Supérieure, ENS, Université PSL, CNRS,Sorbonne Université, Université de Paris, F-75005 Paris, France.

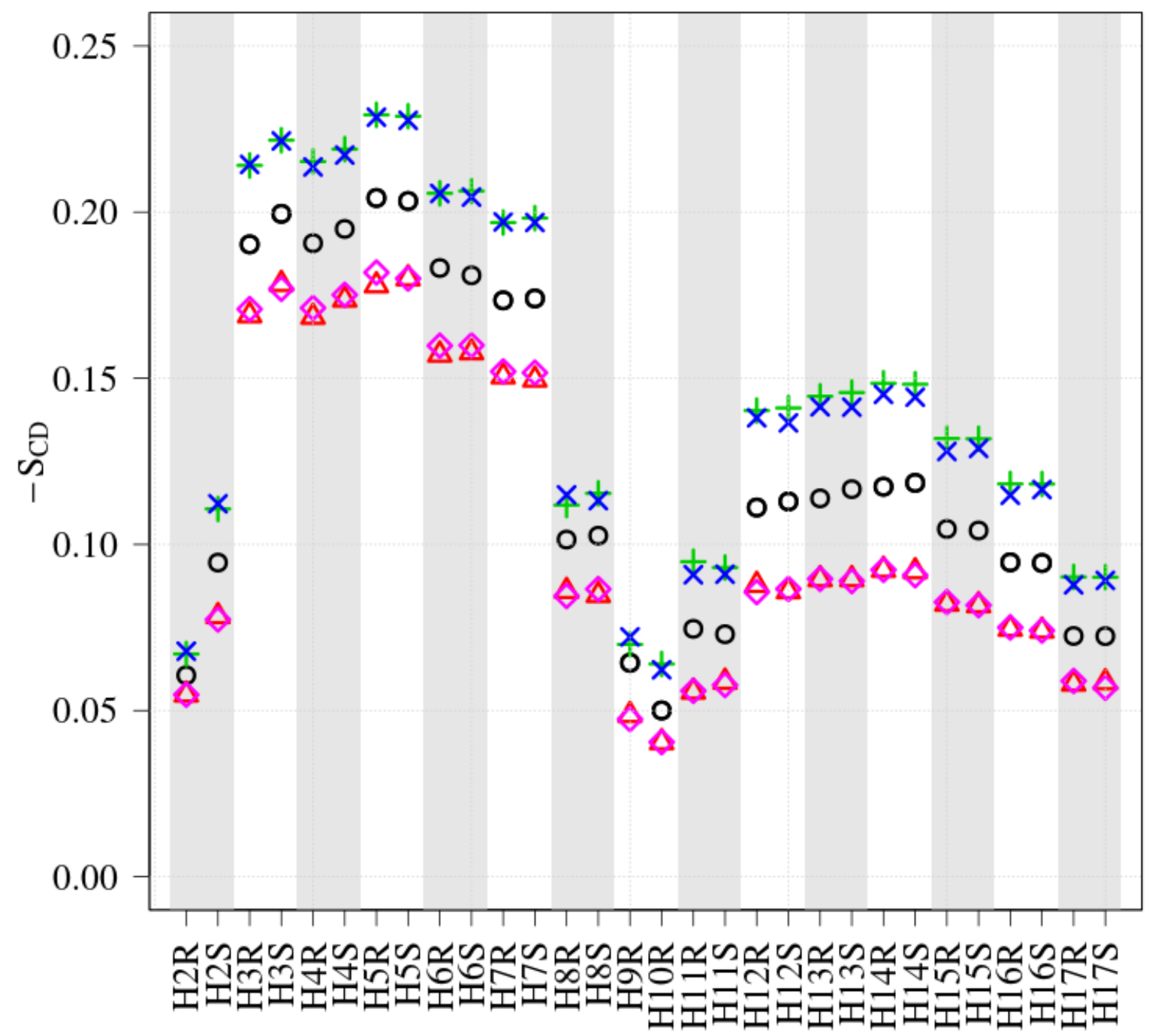

Figure 1S. Order parameter for each hydrogen atoms of one of the sn-2 DOPC acyl chain. The symbols correspond to the case where a uniform pressure of $0.1 \mathrm{MPa}$ was applied $(\mathrm{O})$, when a pressure of $2.4 \mathrm{MPa}(\diamond)$ or a $-2.4 \mathrm{MPa}(+)$ was applied perpendicularly to the membrane surface, and when a pressure of $2.4 \mathrm{MPa}(\times)$ or $-2.4 \mathrm{MPa}(\triangle)$ was applied laterally to the membrane 


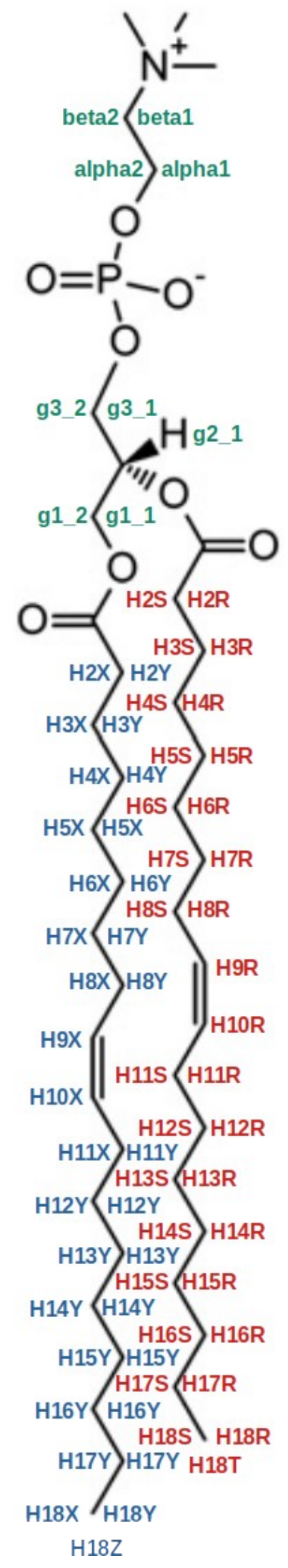

Figure 2S. Chemical structure of a DOPC lipid with the displayed labels used in the $x$-axis of the figures displaying order parameters (i.e. Fig. 5 and 1S). The green labels are for the hydrogen of the DOPC polar head, the blue and red labels are for, respectively, the sn-1 and sn-2 DOPC acyl chains. 


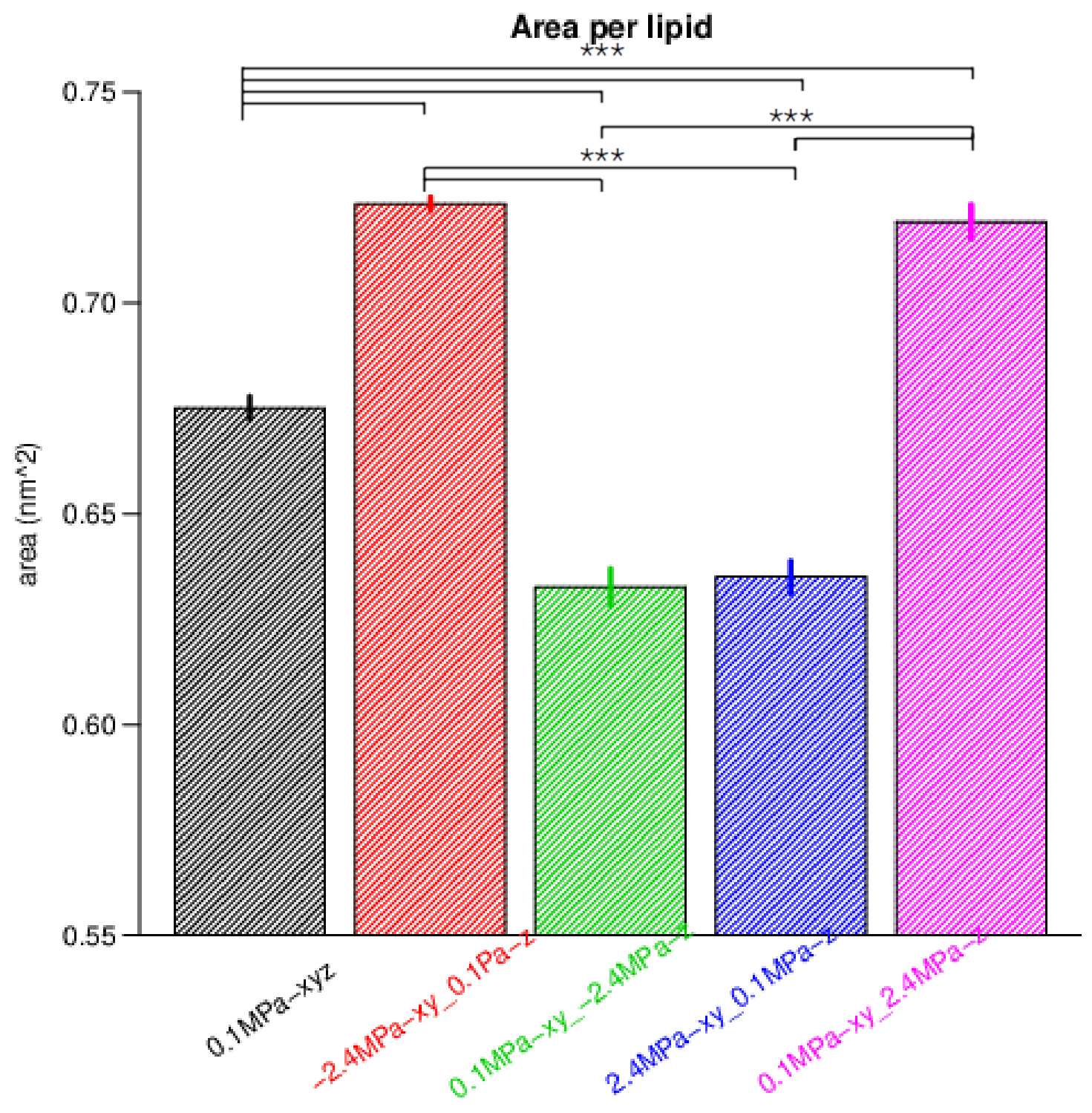

Figure 3S. Bar plot displaying the area per lipid $a$ derived from small size membrane simulations. The bars correspond to the case where a uniform compression of $0.1 \mathrm{MPa}$ was applied in all directions (black), when a pressure of 2.4 MPa (magenta) or a -2.4 MPa (green) was applied perpendicularly to the membrane surface, and when a pressure of 2.4 MPa (blue) or of $-2.4 \mathrm{MPa}$ (red) was applied laterally to the membrane 


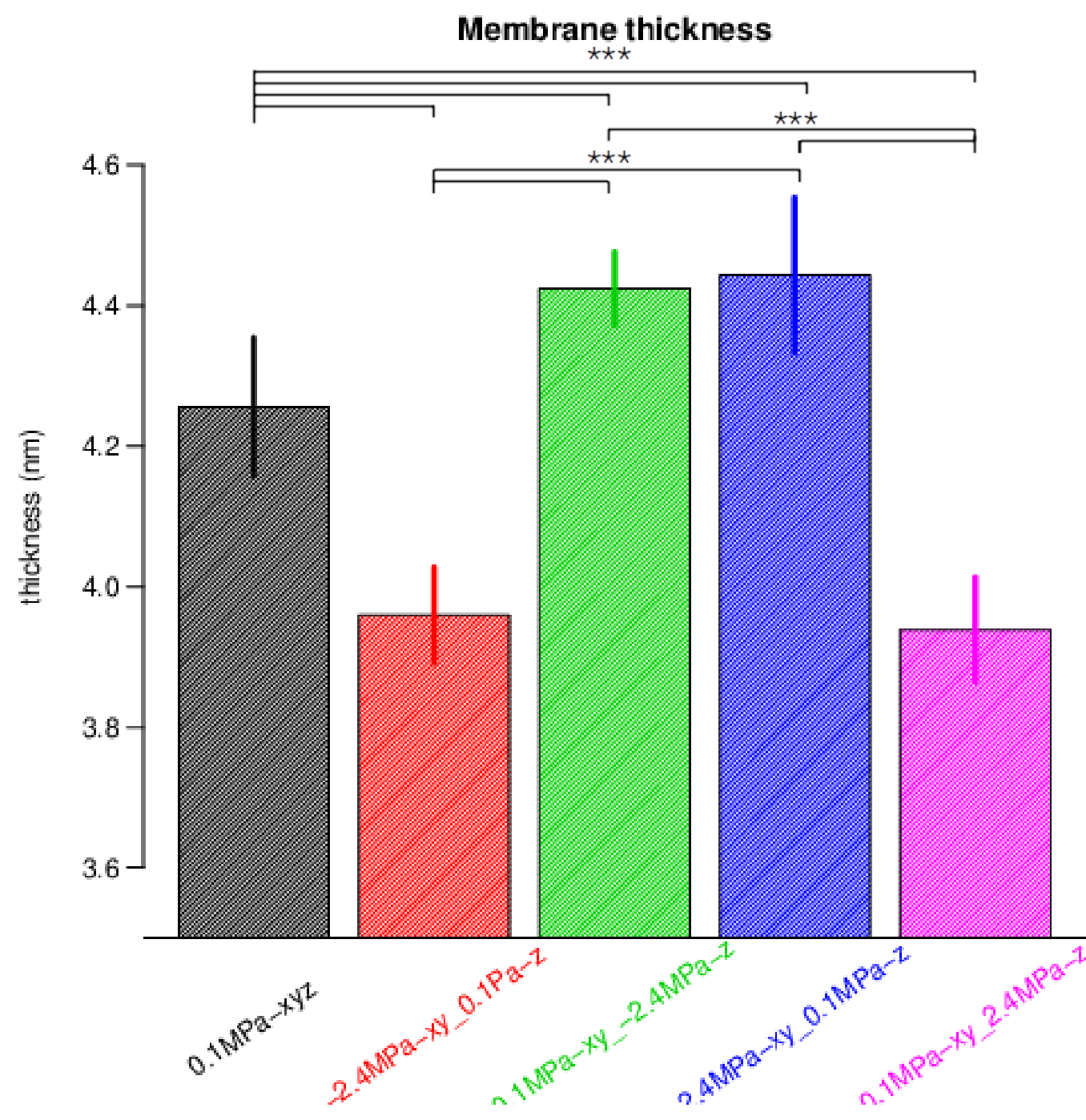

Figure 4S. Bar plot displaying the membrane thickness $d$ derived from small size membrane simulations. The bars correspond to the case where a uniform compression of $0.1 \mathrm{MPa}$ was applied in all directions (black), when a pressure of 2.4 MPa (magenta) or a -2.4 MPa (green) was applied perpendicularly to the membrane surface, and when a pressure of $2.4 \mathrm{MPa}$ (blue) or of $-2.4 \mathrm{MPa}$ (red) was applied laterally to the membrane 


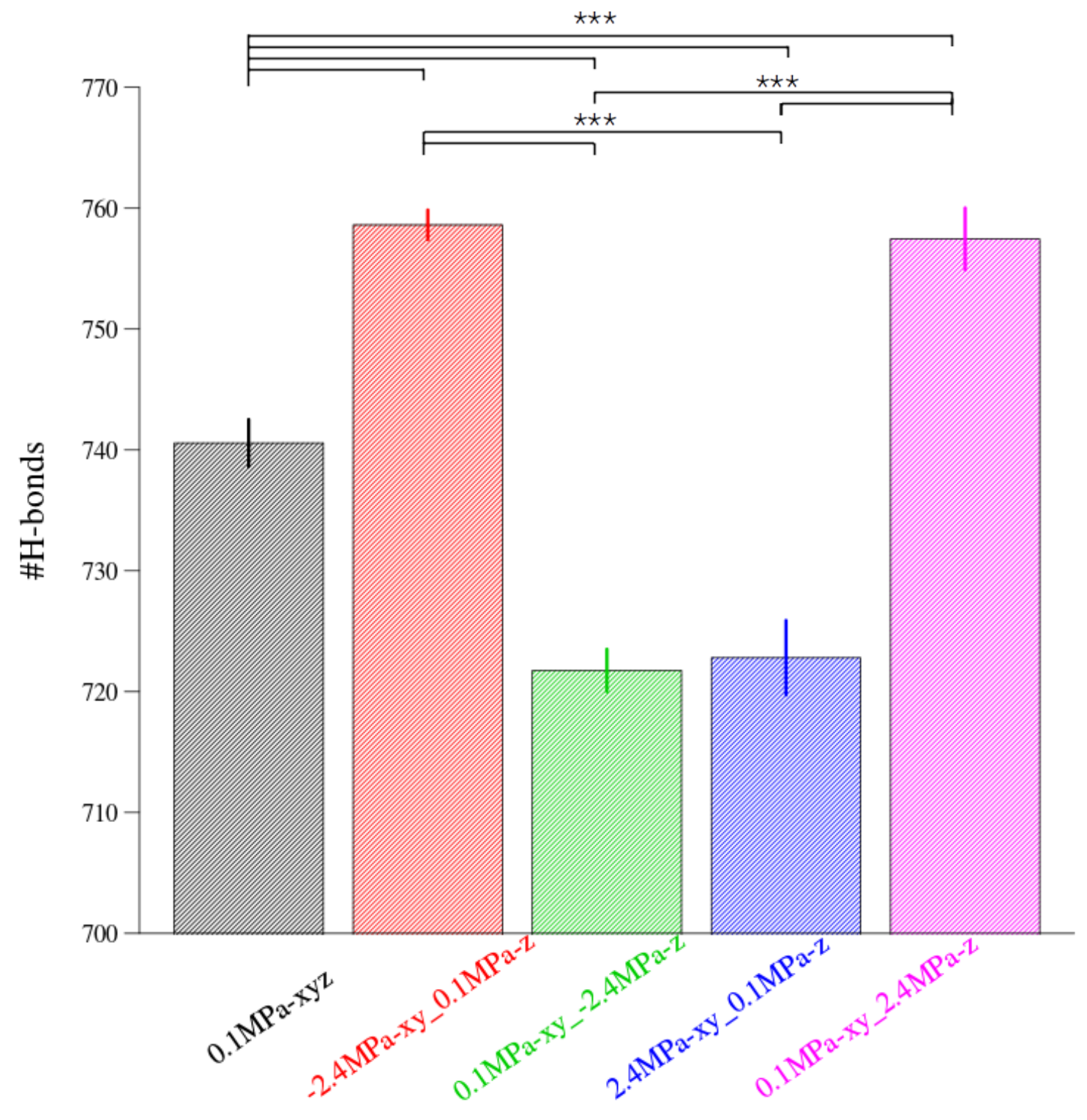

Figure 5S. Bar plot displaying the number of hydrogen bonds between DOPC lipid and water molecules as derived from small size membrane simulations. The bars correspond to the case where a uniform compression of 0.1 MPa was applied in all directions (black), when a pressure of 2.4 MPa (magenta) or a -2.4 MPa (green) was applied perpendicularly to the membrane surface, and when a pressure of 2.4 MPa (blue) or of -2.4 MPa (red) was applied laterally to the membrane 


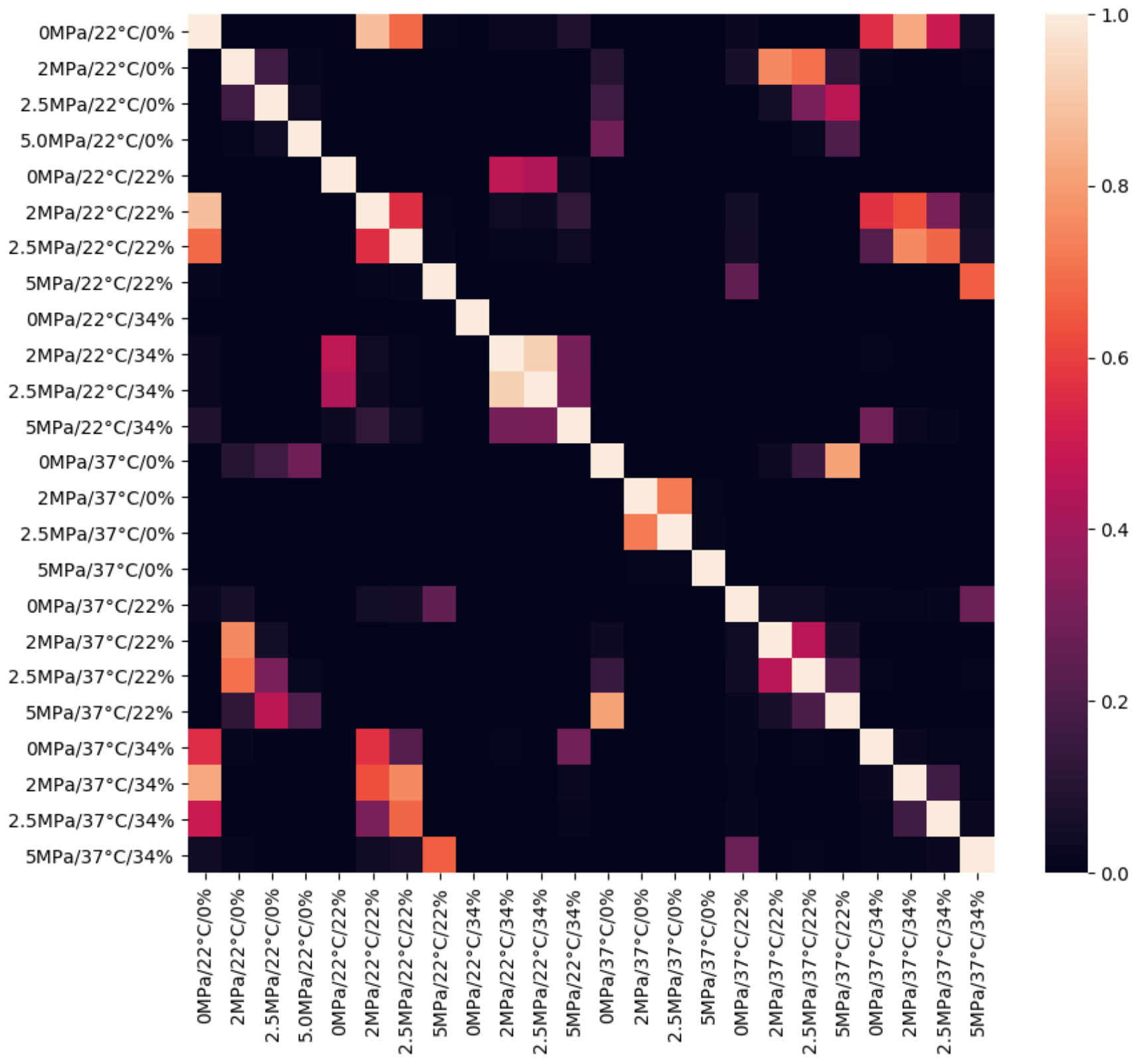

Figure 6S. The figure is a heatmap of p-values that indicates if the points displayed in the top figure of Fig . 3 were significantly different (in black) from each other. Each point is characterized by the tuple (pressure/temperature/cholesterol fraction). 


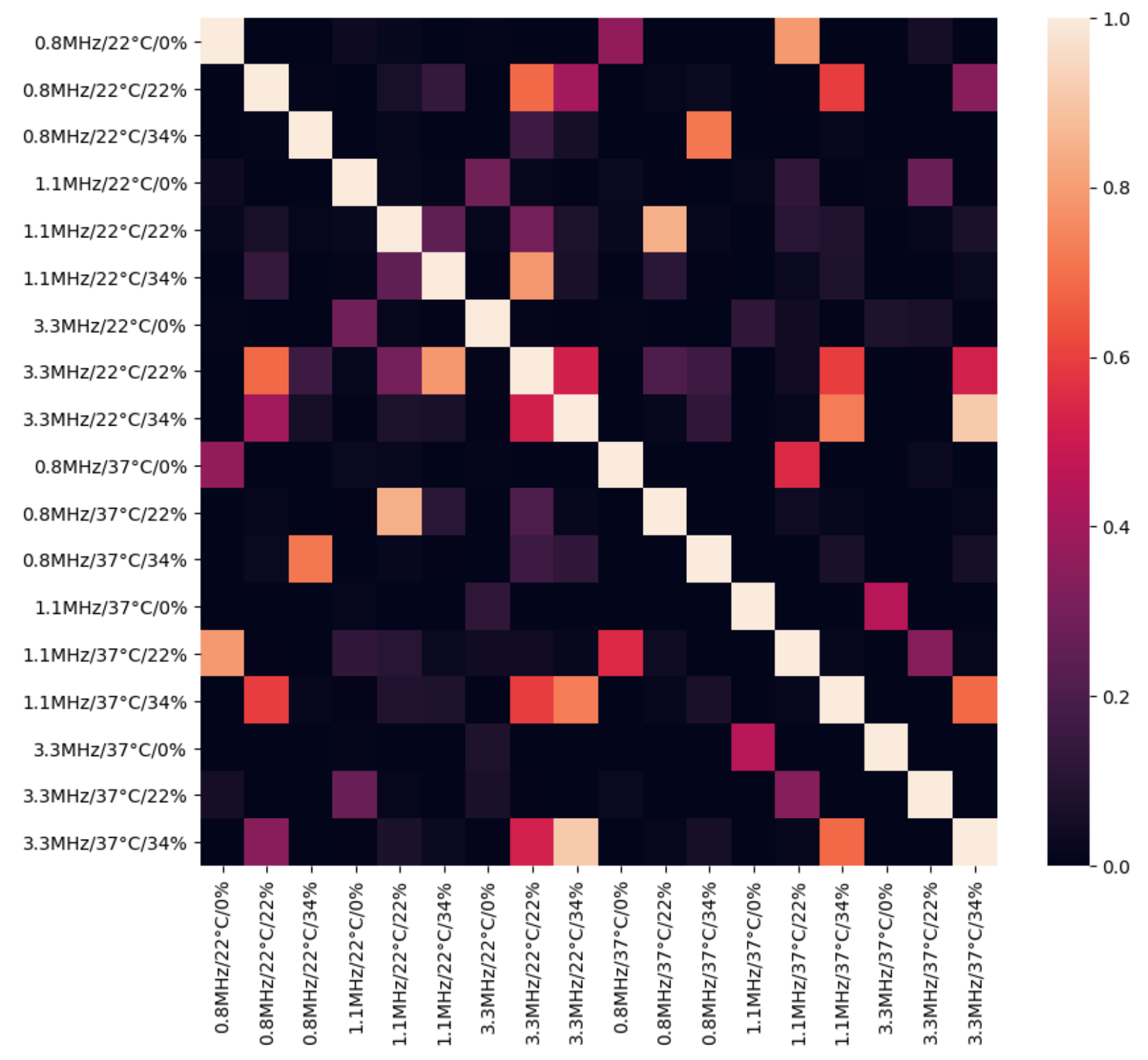

Figure 7S. The figure is a heatmap of p-values that indicates if the points displayed in the bottom figure of Fig . 3 were significantly different (in black) from each other. Each point is characterized by the tuple (pressure/temperature/cholesterol fraction). 


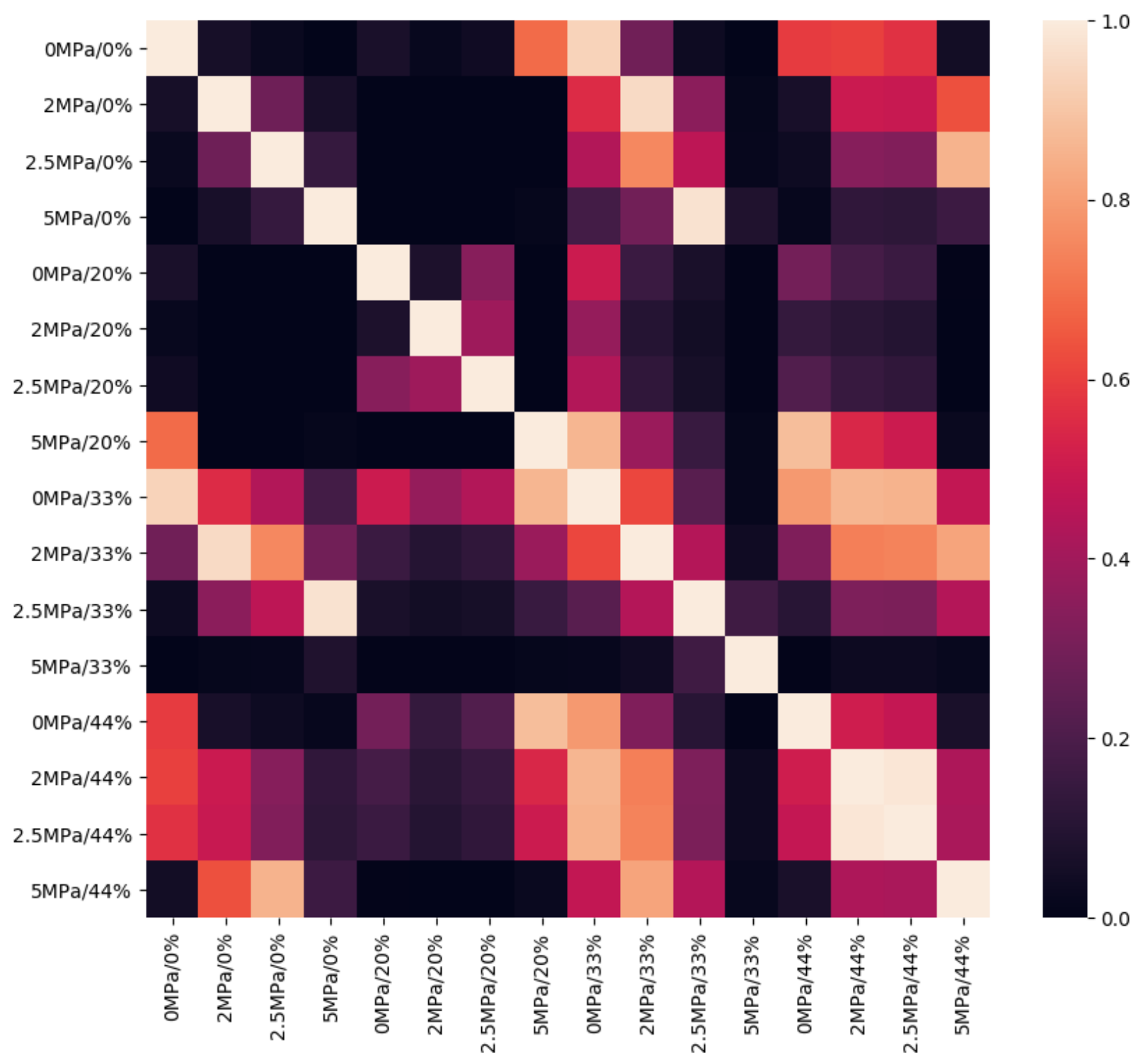

Figure 8S. The figure is a heatmap of p-values that indicates if the points displayed in the top figure of Fig . 4 were significantly different (in black) from each other. Each point is characterized by the tuple (pressure/temperature/cholesterol fraction). 


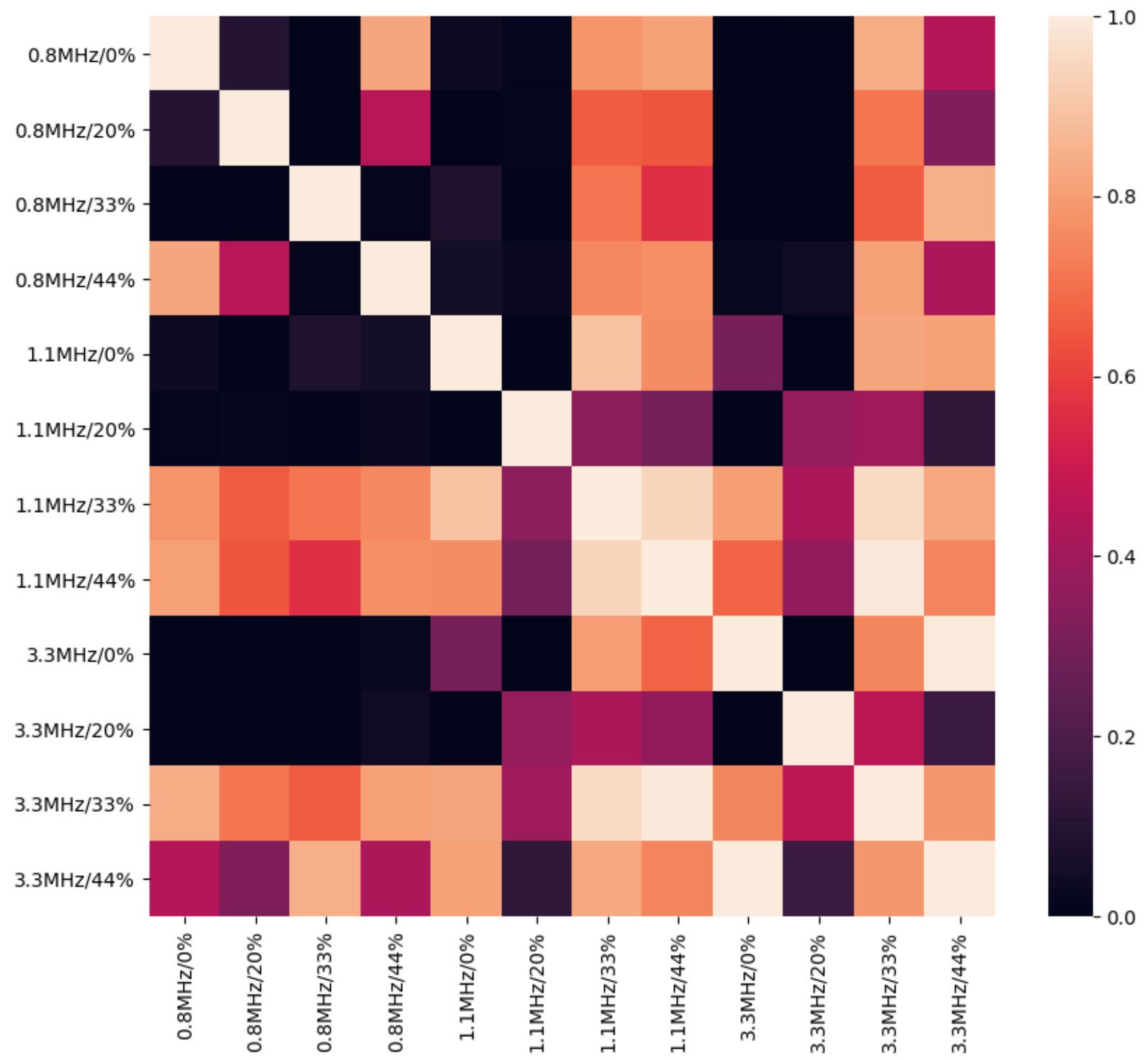

Figure 9S. The figure is a heatmap of p-values that indicates if the points displayed in the bottom figure of Fig . 4 were significantly different (in black) from each other. Each point is characterized by the tuple (pressure/temperature/cholesterol fraction). 

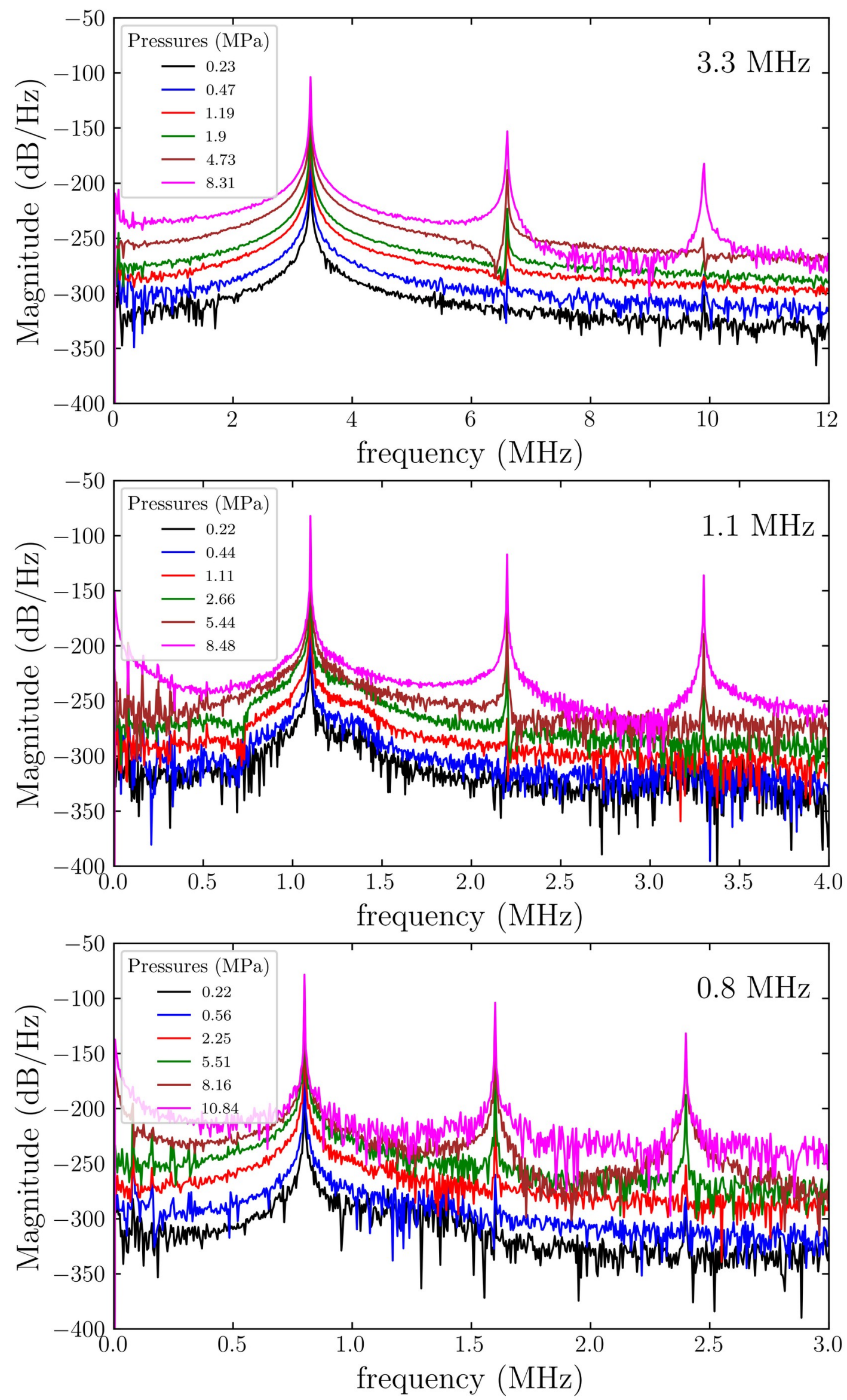

Figure 10S. Power spectrum densities of acoustic signals recorded in water by the needle hydrophone at the focus of the transducer for various peak-to-peak pressures and frequencies. 